\title{
Estimativa da produtividade de trigo em função da adubação nitrogenada utilizando modelagem neuro fuzzy
}

\author{
Aldo A. V. da Silva ${ }^{1}$ Inara A. F. Silva ${ }^{2}$, Marcelo C. M. Teixeira Filho ${ }^{3}$ \\ Salatiér Buzetti ${ }^{4} \&$ Marcelo C. M. Teixeira ${ }^{5}$ \\ ${ }^{1}$ DAI/IFMT, Cuiabá, MT. E-mail: aldovieira@hotmail.com \\ 2 DAI/IFMT, Cuiabá, MT. Email: inaraferrer@gmail.com \\ ${ }^{3}$ DEFERS/FEIS/UNESP, Ilha Solteira, SP. E-mail: mcmteixeirafilho@agr.feis.unesp.br (Autor correspondente) \\ ${ }^{4}$ DEFERS/FEIS/UNESP, Ilha Solteira, SP. E-mail: sbuzetti@agr.feis.unesp.br . Bolsista PQ \\ ${ }^{5}$ DEE/FEIS/UNESP, Ilha Solteira, SP. E-mail: marcelo@dee.feis.unesp.br . Bolsista PQ
}

\section{Palavras-chave:}

Triticum aestivum $\mathrm{L}$.

nitrogênio

redes neurais

ANFIS

sistemas híbridos

\begin{abstract}
R E S U M O
Atualmente, novas técnicas de processamento de dados, tais como redes neurais, lógica nebulosa (fuzzy) e sistemas híbridos, são utilizadas para elaborar modelos de predição em sistemas complexos e estimar parâmetros desejados. Neste artigo investigou-se a habilidade de se desenvolver um modelo de inferência adaptativo neuro fuzzy para estimação da produtividade de trigo utilizando-se uma base de dados da combinação dos seguintes tratamentos: cinco doses de N (0,50,100, 150 e $\left.200 \mathrm{~kg} \mathrm{ha}^{-1}\right)$; três fontes (Entec, sulfato de amônio e ureia); duas épocas de aplicação de N (na semeadura ou em cobertura) e dois cultivares de trigo (E21 e IAC 370), avaliados durante dois anos, em Selvíria, MS. Através dos dados de entrada e saída o sistema de inferência neuro fuzzy adaptativo apreende e posteriormente pode estimar um novo valor de produção de trigo com base em doses diferenciadas de N. O erro de predição da produtividade de trigo em função das cinco doses de N, obtido com o sistema neuro fuzzy, foi menor que o valor obtido utilizando-se uma aproximação quadrática. Os resultados mostraram que o sistema neuro fuzzy é viável para desenvolver um modelo de predição visando estimar a produtividade de trigo em função da dose de $\mathrm{N}$.
\end{abstract}

Key words:

Triticum aestivum $\mathrm{L}$. nitrogen neural networks ANFIS

hybrid systems

\section{Estimate of wheat grain yield as function of nitrogen fertilization using neuro fuzzy modeling}

\begin{abstract}
A B S T R A C T
Currently new techniques for data processing, such as neural networks, fuzzy logic and hybrid systems are used to develop predictive models of complex systems and to estimate the desired parameters. In this article the use of an adaptive neuro fuzzy inference system was investigated to estimate the productivity of wheat, using a database of combination of the following treatments: five $\mathrm{N}$ doses (0, 50, 100, 150 and $200 \mathrm{~kg} \mathrm{ha}^{-1}$ ), three sources (Entec, ammonium sulfate and urea), two application times of $\mathrm{N}$ (at sowing or at side-dressing) and two wheat cultivars (IAC 370 and E21), that were evaluated during two years in Selvíria, Mato Grosso do Sul, Brazil. Through the input and output data, the system of adaptive neuro fuzzy inference learns, and then can estimate a new value of wheat yield with different $\mathrm{N}$ doses. The productivity prediciton error of wheat in function of five $\mathrm{N}$ doses, using a neuro fuzzy system, was smaller than that one obtained with a quadratic approximation. The results show that the neuro fuzzy system is a viable prediction model for estimating the wheat yield in function of $\mathrm{N}$ doses.
\end{abstract}

\section{INTRODUÇÃo}

As condições de solo, clima e topografia, favoráveis ao cultivo de trigo, tanto de sequeiro como irrigado em épocas e altitudes definidas pela pesquisa, fazem do Brasil Central uma região de enorme potencial para a expansão desta cultura com a perspectiva de propiciar, a médio prazo, a autossuficiência na produção nacional. Outrossim, a inserção do trigo no Cerrado contribui para diversificar os sistemas produtivos regionais agregando elementos para a sustentabilidade de produção nesse ecossistema brasileiro (RCCBPT, 2005).
A utilização de cultivares de trigo de alto potencial produtivo de grãos e a adubação nitrogenada são essenciais para a obtenção de altas produtividades porém esta última requer cuidados em seu manuseio, tanto no que concerne à época de aplicação quanto às doses aplicadas. Pequenas doses limitam a produtividade e altas doses podem levar ao acamamento dificultando, assim, a colheita e resultando em queda de produtividade. Além disto, podem trazer prejuízo ao ambiente em função da lixiviação de nitrato para lençois de água e prejuízo para o produtor, com gasto desnecessário com a compra do adubo nitrogenado (Teixeira Filho et al., 
2010); todavia, os estudos sobre a resposta da produtividade do trigo à adubação nitrogenada são insuficientes e devem ser estudados em condições específicas de ambiente como a região dos cerrados de baixa altitude, onde há necessidade do uso da irrigação.

Tradicionalmente, nas pesquisas realizadas na área de ciências agrárias os dados obtidos em campo ou em laboratório são analisados estatisticamente, submetidos à análise de variância, com as médias sendo comparadas sobremaneira pelo teste de Tukey a 0,05 de probabilidade para variáveis qualitativas como, por exemplo, para o efeito de fontes de $\mathrm{N}$, de épocas de aplicação e de cultivares. Para o efeito de doses de $\mathrm{N}$ ou de outro nutriente que são variáveis quantitativas, as médias são ajustadas às equações de regressão, podendo estimar qual é a melhor dose para se obter a máxima produtividade.

Recentemente, novas técnicas, como redes neurais (Bucene \& Rodrigues, 2004; Alvarez, 2009; Kashaninejad et al., 2009), sistemas de inferência nebulosos (fuzzy) (Carvalho et al., 2009), computação evolucionária e sistemas híbridos (Schultz \& Wieland, 1997) têm sido empregadas para desenvolver modelos de predição e estimar parâmetros. Essas técnicas têm utilidade em diversas áreas de pesquisa, porque são adequadas para a análise de sistemas com incertezas, sendo usadas como alternativas aos métodos estatísticos (Yilmaz \& Kaynar, 2011).

Baseados no pioneirismo de Zadeh (1965) os sistemas nebulosos (fuzzy) podem processar, de forma conveniente, informações imprecisas mas não adquirem automaticamente as regras de que necessitam para produzir os resultados almejados. As regras devem ser elaboradas por especialistas que fornecem sua experiência profissional para a elaboração de um sistema de inferência baseado em regras do tipo "Se < condição $>$ Então $<$ resultado>", usadas para realizar a análise desejada.

As redes neurais artificiais são modelos computacionais inspirados no sistema nervoso dos seres vivos, com capacidade de aquisição e manutenção do conhecimento baseado em informações fornecidas pelo ser humano (Silva et al., 2010).

Os modelos fuzzy e as redes neurais são técnicas que possibilitam a descrição de sistemas complexos com comportamentos não lineares. Assim, a integração dos dois sistemas, fuzzy e redes neurais, formando os sistemas híbridos ou neuro fuzzy, visa à obtenção de um sistema mais eficiente na predição, aprendizado, estimação de parâmetros e na capacidade de generalização.

A aplicação do sistema de inferência híbrido ou neuro fuzzy na modelagem e predição, vem sendo amplamente explorada na agricultura. Em agronomia e biologia, pesquisadores e engenheiros têm desenvolvido métodos utilizando lógica nebulosa, redes neurais artificiais, algoritmos genéticos, árvores de decisão e máquinas de vetor de suporte para estudos do solo e regimes da água, relacionados com o crescimento de culturas, para o processamento de alimentos e para a tomada de decisão na agricultura de precisão (Huang et al., 2010). O modelo neuro fuzzy já foi aplicado na análise da auto-hidrólise da Paulownia spp. para fabricação de pasta de celulose (Zamudio et al., 2011), como metodologia para descrever o grau de severidade da ferrugem (Phakopsora pachyrhizi) da soja (Alves et al., 2011) e na predição da absorção de água pelo solo, comparando-o com o modelo estatístico de regressão múltipla (Yilmaz \& Kaynar, 2011).

Objetivou-se, neste trabalho, investigar a possibilidade da obtenção de um modelo de inferência neuro fuzzy, que permita estimar a produtividade de grãos de trigo em função da adubação nitrogenada (com base em dados experimentais de uma produção) e compará-lo com uma aproximação quadrática, usualmente utilizada.

\section{Material e Métodos}

O experimento foi conduzido em 2006 e 2007, em área experimental pertencente à Faculdade de Engenharia da Universidade Estadual Paulista, em Selvíria, MS, a $20^{\circ} 22^{\prime}$ $\mathrm{S}, 51^{\circ} 22^{\prime} \mathrm{W}$ e altitude de $335 \mathrm{~m}$. O solo é classificado como Latossolo Vermelho distrófico, de textura argilosa (Santos et al., 2006), com valores de granulometria de 420,50 e $530 \mathrm{~g} \mathrm{~kg}^{-1}$ de areia, silte e argila, respectivamente. Este solo foi ocupado originalmente por vegetação de cerrado e é cultivado por culturas anuais há mais de 25 anos. O clima da região é Aw, de acordo com Köppen, definido como tropical úmido, com estação chuvosa no verão e seca no inverno. As médias anuais de temperatura e precipitação pluvial são de $23,5^{\circ} \mathrm{C}$ e 1.370 $\mathrm{mm}$ e a umidade relativa do ar fica entre 70 e $80 \%$. Os valores diários de temperatura média e umidade relativa do ar e os totais diários de precipitação pluvial durante o período de condução do experimento, estão apresentados na Figura 1.

Os atributos químicos do solo da área experimental na camada de 0-0, $20 \mathrm{~m}$ de profundidade foram determinados antes da instalação do experimento, segundo metodologia proposta por van Raij et al. (2001), e tiveram os seguintes

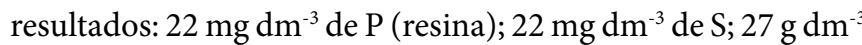
de matéria orgânica; 5,9 de pH em $\mathrm{CaCl}_{2} ; 3,3,47,0,16,0$ e 28,0 $\mathrm{mmol}_{\mathrm{c}} \mathrm{dm}^{-3} \mathrm{de} \mathrm{K}, \mathrm{Ca}, \mathrm{Mg}, \mathrm{H}+\mathrm{Al}$, respectivamente e $70 \%$ de saturação por bases.

O delineamento experimental utilizado foi o de blocos ao acaso com quatro repetições em esquema fatorial $5 \times 3 \times 2 \times$ 2. Os tratamentos consistiram da combinação de cinco doses de N: 0, 50, 100, 150 e $200 \mathrm{~kg} \mathrm{ha}^{-1}$; três fontes: Entec, sulfato de amônio (SA) e ureia; duas épocas de aplicação totalmente na semeadura, ao lado das linhas, e em cobertura nas entrelinhas, além de dois cultivares de trigo: E 21 e IAC 370; as dimensões das parcelas foram de $5 \mathrm{~m}$ de comprimento, com cinco linhas espaçadas $0,17 \mathrm{~m}$ e 80 sementes por metro, nos dois anos agrícolas; enfim, a área útil da parcela com exclusão das bordaduras, foi de $2,55 \mathrm{~m}^{2}$.

Os tratamentos foram instalados em local onde o sistema plantio direto havia sido implantado há oito anos. Durante o verão a área foi cultivada com milho, tanto em 2006 quanto em 2007. Antes da semeadura do trigo a área foi dessecada com o herbicida glifosato (1.500 $\mathrm{g} \mathrm{ha}^{-1}$ do i.a.). A semeadura 

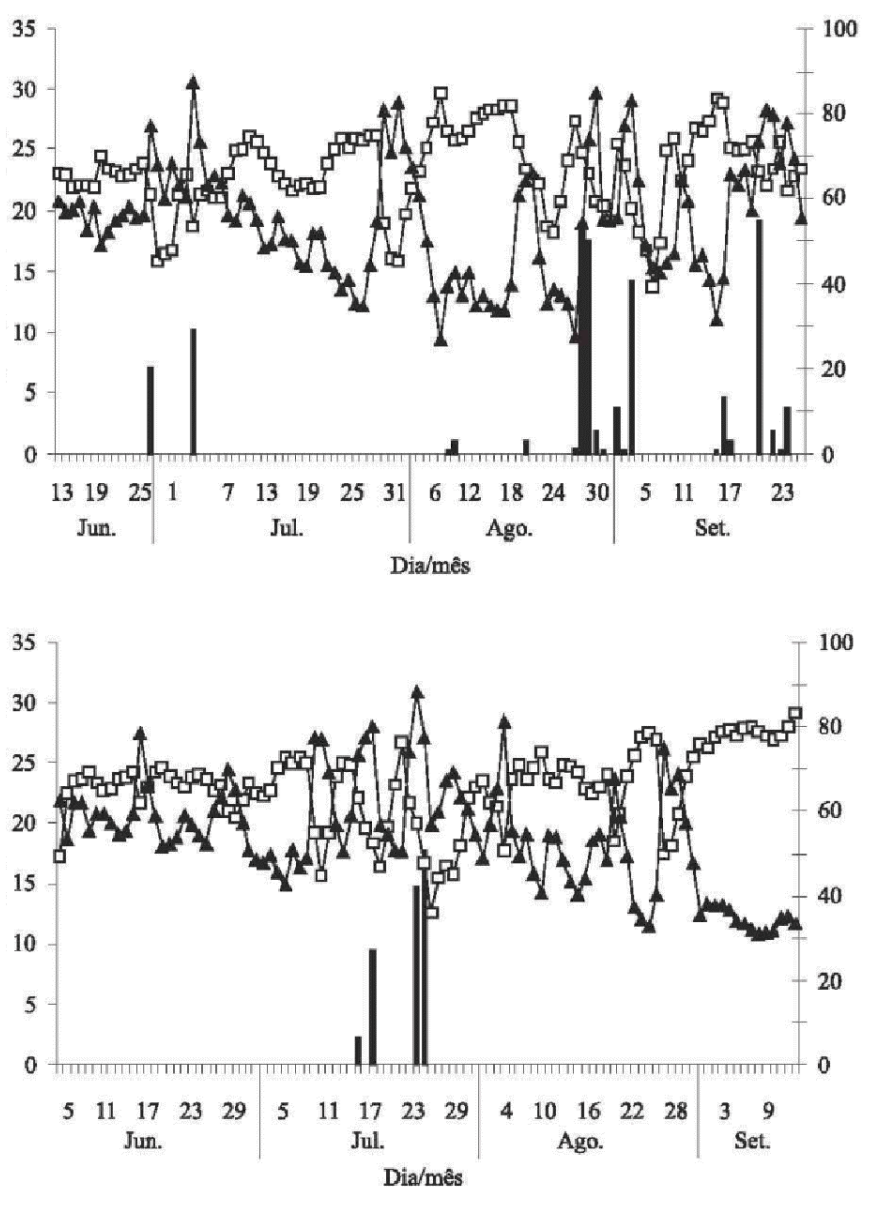

Precipitação(mm) $\quad \rightarrow-$ Temp. media $\left({ }^{\circ} \mathrm{C}\right) \quad$ —UR (\%)

Figura 1. Precipitação pluvial, temperatura média e umidade relativa do ar (UR) diárias, durante a condução do experimento, em 2006 (A) e 2007 (B)

foi realizada mecanicamente nos dias 13 e 5 de junho de 2006 e 2007, respectivamente; em seguida, a área foi irrigada por aspersão, por meio de pivô central, com lâmina de água de aproximadamente $14 \mathrm{~mm}$, para minimizar as perdas de $\mathrm{N}$ por volatilização da amônia e promover a germinação das sementes. As plântulas emergiram cinco dias após a semeadura.

Com base nas características químicas do solo da área experimental calculou-se a adubação química básica no sulco de semeadura, constante para todos os tratamentos, que foi de: 10 , 75 e $25 \mathrm{~kg} \mathrm{ha}^{-1}$ de N, $\mathrm{P}_{2} \mathrm{O}_{5}$ e $\mathrm{K}_{2} \mathrm{O}$, respectivamente, em ambos os anos de cultivo. Para a adubação na semeadura foram aplicados os seguintes fertilizantes: ureia, superfosfato simples e cloreto de potássio. A adubação nitrogenada de cobertura foi realizada nas entrelinhas das parcelas, sem incorporação ao solo, aos 40 dias após a emergência das plantas, no estádio de emborrachamento da cultura; após esta adubação a área foi irrigada por aspersão com uma lâmina de água de aproximadamente $14 \mathrm{~mm}$; esta operação é comum nos sistemas de produção de grãos irrigados quando se aplica a ureia.

O fornecimento de água foi efetuado a cada três dias ou quando necessário, por meio de um sistema fixo de irrigação por aspersão do tipo pivô central. O manejo de plantas daninhas foi efetuado com a aplicação do herbicida metsulfuron methyl (3,0 $\mathrm{g} \mathrm{ha}^{-1}$ do i.a.), em pós-emergência, em 2006 e 2007. A colheita do trigo foi realizada manualmente nas três linhas centrais de cada parcela, em 2006 e 2007, aos 105 e 100 dias após a emergência das plantas, respectivamente, quando $90 \%$ das espigas apresentavam coloração típica de grãos maduros. O material colhido foi submetido a secagem a pleno sol e posteriormente trilhado. Após a trilhagem mecânica os grãos foram quantificados e os dados foram transformados em quilograma por hectare, para 13\% (base úmida).

Primeiro, os dados foram submetidos à análise de variância e as médias foram comparadas pelo teste de Tukey, a 5\% de probabilidade quanto ao efeito das fontes de $\mathrm{N}$, de épocas de aplicação e de cultivares e ajustadas às equações de regressão do efeito das doses de N. Para a análise estatística foi utilizado o programa SISVAR (Ferreira, 2008).

Através deste experimento realizado em Selvíria, MS, no período de dois anos, selecionou-se o conjunto de dados; esses dados foram utilizados pela abordagem neuro fuzzy, para estimar a produção de trigo em função da adubação nitrogenada, com cinco amostras referentes ao ano de 2006 e cinco amostras do ano de 2007. Os valores médios de produtividade do trigo referente às doses $0,50,100,150$ e $200 \mathrm{~kg} \mathrm{ha}^{-1}$, foram utilizados para treinamento do sistema.

Para o treinamento, teste e consequente elaboração do modelo, utilizou-se um dos primeiros sistemas híbridos propostos: o sistema de inferência adaptativo neuro fuzzy (ANFIS).

O sistema de inferência neuro fuzzy adaptativo é implementado através da função anfisedit da toolbox fuzzy logic do Matlab (Mathworks-Fuzzy Logic Toolbox, 2002).

O ANFIS é utilizado em aplicações de previsão e aproximação de funções e emprega, como sistema de inferência, um modelo fuzzy Takagi-Sugeno (Jang \& Sun, 1995), de ordem zero (constante) ou ordem 1 (linear) (Tanscheit et al., 2007) que possui uma única saída; neste caso, a estimação da produção do trigo.

Serão apresentados, a seguir, alguns detalhes relativos à estrutura do sistema de inferência neuro fuzzy adaptativo e sua aplicação na estimação da produtividade do trigo.

Considere $\mathrm{X}$ um conjunto e $\mathrm{x}$ um elemento genérico de $\mathrm{X}$. Um conjunto clássico $\mathrm{A} \subseteq \mathrm{X}$ é definido como uma coleção de elementos $\mathrm{x} \in \mathrm{X}$ tal que cada $\mathrm{x}$ pode ou não pertencer a $\mathrm{X}$. Por outro lado, um conjunto fuzzy $\mathrm{A}$, referindo-se às doses de $\mathrm{N}$ $\left(\mathrm{kg} \mathrm{ha}^{-1}\right)$, pode ser definido como um conjunto ordenado de pares sendo que $\mu_{\mathrm{A}}(\mathrm{x})$ denota a função de pertinência $(\mathrm{mf})$ para o conjunto $A$, com valores entre 0 e 1 , revelando o grau de pertinência do elemento $\mathrm{x}$ no conjunto A (Zadeh, 1968). Então, um conjunto fuzzy A em X é definido como um conjunto ordenado de pares:

$$
\mathrm{A}=\left\{\left(\mathrm{x}, \mu_{\mathrm{A}}(\mathrm{x})\right) / \mathrm{x} \in \mathrm{X}\right\}
$$

em que os elementos $\mathrm{x}$ representam as doses de $\mathrm{N}$. 
Para o modelo de inferência Takagi-Sugeno utilizado pelo ANFIS, a composição comum das regras fuzzy com uma entrada é a seguinte (Jang, 1993):

$$
\text { Regra i: Se x é } A_{i} \text {, então } f_{i}=p_{i} x+g_{i}, i=1,2,3,4,5
$$

sendo que $\mathrm{x}$ pertence a $\mathrm{X}$, que é o conjunto fuzzy antecedente com os valores de doses de nitrogênio aplicadas, enquanto $\mathrm{f}(\mathrm{x})$ é uma função polinomial da variável de entrada $\mathrm{x}$.

A descrição do sistema de inferência neuro fuzzy, representado na Figura 2, tem a estrutura da rede para a situação de uma entrada $\{x\}$, particionada em cinco conjuntos fuzzy $A_{i}$, que correspondem aos valores das doses: 0, 50, 100, 150 e 200 $\mathrm{kg} \mathrm{ha}^{-1}$.

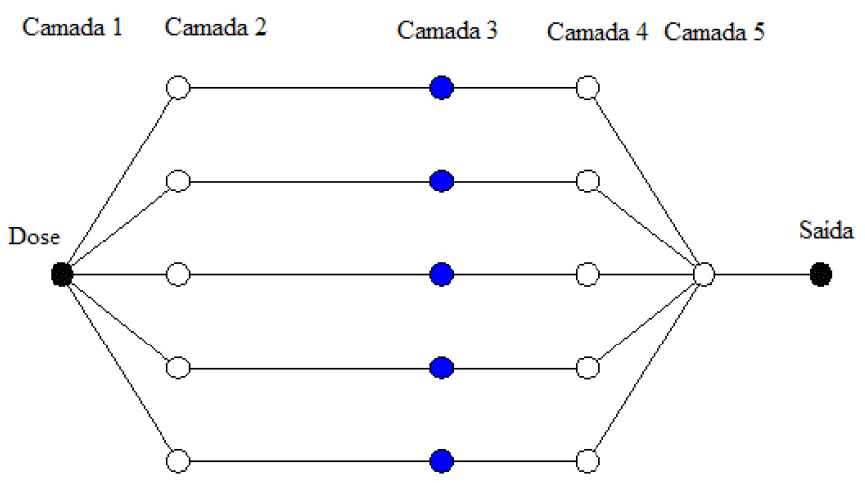

Figura 2. Arquitetura ANFIS de uma entrada e uma saída com cinco regras no sistema de estimação da produção do trigo em função da adubação nitrogenada

As etapas de desenvolvimento do sistema de inferência neuro fuzzy adaptativo (ANFIS), para estimar a produtividade de trigo em função da adubação nitrogenada, têm a arquitetura composta de cinco camadas, cada uma com sua finalidade específica (Jang, 1993). São descritas a seguir:

Camada 1: calcula-se o grau de pertinência de cada entrada (x corresponde à dose de nitrogênio), de acordo com a função de pertinência escolhida; neste trabalho escolheu-se a gaussmf como função de pertinência para representar os efeitos das doses na estimação da produtividade de trigo em função da adubação nitrogenada. São cinco funções de pertinência (mf) para os valores das doses, utilizadas para a construção do modelo:

$$
\mathrm{O}_{1, \mathrm{i}}=\mu_{\mathrm{Ai}}(\mathrm{x})=\mathrm{e}^{-\frac{1}{2}\left(\frac{\mathrm{x}-\mathrm{x}_{\mathrm{i}}^{\prime}}{\sigma_{\mathrm{i}}}\right)^{2}}, \mathrm{i}=1,2,3,4,5
$$

sendo que:

$\left\{\mathrm{x}_{\mathrm{i}}^{\prime}, \sigma_{\mathrm{i}}\right\}, \mathrm{i}=1,2,3,4,5$, representam o centro e a largura da função de pertinência gaussiana durante o treinamento híbrido e x é a entrada do sistema

Camada 2: Cada nó desta camada corresponde a uma regra e calcula com que grau o consequente da regra é atendido; nesta camada os neurônios executam a operação de produto
(T-norm), ou seja, multiplicam todos os sinais de entrada e os enviam para a saída:

$$
\mathrm{O}_{2, \mathrm{i}}=\mathrm{w}_{\mathrm{i}}=\prod_{\mathrm{i}=1}^{5} \mu_{\mathrm{Ai}}(\mathrm{x}), \mathrm{i}=1,2,3,4,5
$$

Camada 3: Cada nó calcula a razão entre o valor de disparo da regra e a soma dos valores de todas as regras. A saída é a normalização dos valores, utilizada como um préprocessamento para a defuzzyficação. As saídas dos nós desta camada são:

$$
\mathrm{O}_{3, \mathrm{i}}=\overline{\mathrm{w}_{\mathrm{i}}}=\frac{\mathrm{w}_{\mathrm{i}}}{\sum_{\mathrm{i}}^{5} \mathrm{w}_{\mathrm{i}}}, \mathrm{i}=1,2,3,4,5
$$

Camada 4: Nesta camada as saídas dos neurônios são calculadas pelo produto entre os valores normalizados e o valor do consequente da regra em $\mathrm{w}_{\mathrm{i}}$. Os valores de saída de cada nó desta camada são dados por:

$$
\mathrm{O}_{4, \mathrm{i}}=\overline{\mathrm{w}_{\mathrm{i}}} \mathrm{f}_{\mathrm{i}}=\overline{\mathrm{w}_{\mathrm{i}}} \times\left(\mathrm{p}_{\mathrm{i}} \mathrm{x}+\mathrm{r}_{\mathrm{i}}\right), \mathrm{i}=1,2,3,4,5
$$

sendo que: os valores de $\mathrm{O}_{4, \mathrm{i}}, \mathrm{i}=1,2,3,4,5$ correspondem aos consequentes Takagi-Sugeno de primeira ordem (combinações lineares das entradas).

Camada 5: O nó desta última camada da arquitetura calcula a saída precisa do sistema e, juntamente com os nós das camadas 3 e 4 , promove a defuzzyficação ou soma total de todos os sinais de entrada. Sua saída é dada por:

$$
\mathrm{O}_{5, \mathrm{i}}=\sum_{\mathrm{i}} \overline{\mathrm{w}_{\mathrm{i}}} \mathrm{f}_{\mathrm{i}}=\frac{\sum_{\mathrm{i}} \mathrm{w}_{\mathrm{i}} \mathrm{f}_{\mathrm{i}}}{\sum_{\mathrm{i}} \mathrm{w}_{\mathrm{i}}}, \mathrm{i}=1,2,3,4,5
$$

Pela arquitetura ANFIS observa-se que nos valores das premissas a produção total pode ser expressa como a combinação linear dos parâmetros consequentes:

$$
\mathrm{f}=\sum_{\mathrm{i}} \overline{\mathrm{w}_{\mathrm{i}}} \mathrm{f}_{\mathrm{i}}=\sum_{\mathrm{i}} \overline{\mathrm{w}_{\mathrm{i}}}\left(\mathrm{p}_{\mathrm{i}} \mathrm{x}+\mathrm{g}_{\mathrm{i}}\right), \mathrm{i}=1,2,3,4,5
$$

que é uma combinação linear dos parâmetros consequentes $\mathrm{p}_{\mathrm{i}}$.

O ANFIS é um método de aproximação baseado na adaptação de regras, cujo treinamento é usado para "ensinar" o sistema neuro fuzzy na adaptação de seus parâmetros. Este utiliza duas técnicas na atualização de parâmetros (Ghoush, 2008): o backpropagation (gradiente descendente) e o híbrido.

A aprendizagem do treinamento híbrido do ANFIS utilizada neste estudo é a combinação dessas duas técnicas (Ghoush et al., 2008):

a) Para a aprendizagem dos parâmetros dos antecedentes utiliza-se o gradiente descendente da rede neural backpropagation para refinar o erro; 
b) Para a aprendizagem dos parâmetros consequentes, que definem os coeficientes de cada equação de saída, usa-se o método dos mínimos quadrados para identificá-los.

Para determinar e avaliar a performance dos resultados de estimação da produção do trigo utilizou-se, neste artigo, a raiz quadrada do erro médio quadrático (RMSE):

$$
\operatorname{RMSE}=\sqrt{\frac{1}{\mathrm{n}} \sum_{\mathrm{i}=1}^{\mathrm{n}}\left(\mathrm{y}_{\mathrm{i}}-\mathrm{y}_{\mathrm{i}}^{\prime}\right)^{2}}
$$

sendo que:

$y_{i}$ e $y_{i}^{\prime}$ - são o vetor desejado e vetor observado, respectivamente

n - o número de pares de entrada-saída do vetor usado no treinamento

Uma estimação perfeita é obtida se o RMSE é igual a zero.

Para efeito deste estudo consideram-se, como entrada, as doses de nitrogênio e como saída os valores médios da produtividade de grãos de trigo (que correspondem à variável $\left.y_{i}^{\prime}\right)$. O objetivo foi comparar as estimativas de produção obtidas com o ANFIS, com os resultados obtidos utilizando-se regressão quadrática.

As variáveis usadas no arquivo de treinamento do referido modelo foram:

- Dados de entrada: Dose de aplicação;

- Dados de saída: Produção média dos grãos de trigo em função da adubação nitrogenada.

Para elaboração do modelo neuro fuzzy, enumeraram-se as fases de:

- Preparação do arquivo de treinamento (Tabelas 1 e 2);

- Carregamento dos arquivos de treinamento;

- Número e tipos de funções de pertinências de entradas e da saída ( 5 funções de pertinência do tipo gaussiana para os valores das doses de entrada);

- Escolheu-se o método para o treinamento da rede neural (método híbrido);

Tabela 1. Dados de treinamento do ano de 2006, valores médios de produtividade de trigo por dose de $\mathrm{N}$

\begin{tabular}{|c|c|}
\hline Doses de N (kg ha-1) & Produtividade de grãos $\left(\mathrm{kg} \mathrm{ha}^{-1}\right)$ \\
\hline 0 & 3029 \\
\hline 50 & 3755 \\
\hline 100 & 3791 \\
\hline 150 & 3690 \\
\hline 200 & 3543 \\
\hline
\end{tabular}

Tabela 2. Dados de treinamento do ano de 2007, valores médios de produtividade de trigo por dose de $\mathrm{N}$

\begin{tabular}{cc}
\hline Doses de N $\left(\mathbf{k g ~ h a}^{-1}\right)$ & Produtividade de grãos $\left(\mathbf{k g ~ h a}^{-1}\right)$ \\
0 & 2537 \\
50 & 4164 \\
00 & 4021 \\
150 & 3858 \\
200 & 3680 \\
\hline
\end{tabular}

- Escolheram-se o erro e a época desejados (critérios de parada do modelo);

- Iniciou-se o treinamento da rede neural com os dados da Tabela 1.

Após estabelecer números e os tipos de funções de pertinência de entrada e escolher a função de saída, a ferramenta computacional, após o treinamento, apresenta os parâmetros da rede ANFIS conforme a Tabela 3.

Tabela 3. Parâmetros do ANFIS após treinamento

\begin{tabular}{lc}
\hline \multicolumn{1}{c}{ Tipo de parâmetro ANFIS } & Valor \\
Funções de pertinência (MF) & Gaussmf \\
Função de Saída (deffuzificação) & Constante \\
Número de nós: & 24 \\
Número de regras fuzzy & 5 \\
Número de parâmetros lineares & 5 \\
Número de parâmetros não lineares & 10 \\
Total de parâmetros & 15 \\
Número de pares de treinamento & 5 \\
Número de pares de validação & 0 \\
\hline
\end{tabular}

A partir da escolha dos vetores de entrada Tabelas 1 e 2, da escolha das funções de pertinência e da seleção do tipo de treinamento da rede (backpropagation ou híbrido), o ANFIS consegue, por intermédio dos algoritmos backpropagation ou híbrido, ajustar os coeficientes dos polinômios e as funções de pertinência das entradas otimizando a inferência sobre a planta representada (Silva et al., 2010).

Com o uso das técnicas de otimização não linear do modelo ANFIS, um sistema de previsão foi implementado utilizandose, como variáveis independentes, as doses aplicadas e, como variáveis dependentes, a produção de trigo, em função da adubação nitrogenada.

\section{Resultados e Discussão}

Com relação aos dados experimentais do trigo, cultivados em Selvíria, MS, observou-se que não houve diferença significativa entre a produtividade de grãos dos cultivares de trigo IAC 370 e E 21, tanto em 2006 como em 2007 (Tabela 4).

As épocas de aplicação também não interferiram na produtividade de grãos devido, provavelmente, ao fato de que o solo onde o experimento foi conduzido tenha apresentado boa fertilidade e drenagem, por ser bastante argiloso e ter apresentou um teor de matéria orgânica $\left(27 \mathrm{~g} \mathrm{dm}^{-3}\right)$ considerado médio (26 a $50 \mathrm{~g} \mathrm{dm}^{-3} \mathrm{de} \mathrm{MO),} \mathrm{segundo} \mathrm{a} \mathrm{CQFS-RS/SC} \mathrm{(2004).}$ Além disto, o trigo foi cultivado num período de seca com pouca chuva e, portanto, a prática da aplicação antecipada do nutriente foi eficaz. Também Ros et al. (2003) verificaram, avaliando a disponibilidade de $\mathrm{N}$ e a produtividade de trigo com métodos de adubação nitrogenada em plantio direto, que a aplicação de $\mathrm{N}$ total na semeadura ou em cobertura não diferiu na produtividade de grãos, considerando a mesma dose.

$\mathrm{Na}$ Tabela 4 verifica-se que não houve diferença entre as fontes de $\mathrm{N}$ para a produtividade de grãos de trigo, em 2006 e 2007. Esta ausência de resposta das fontes de $\mathrm{N}$ ocorreu devido, sem dúvida, ao fato de que logo após a sua aplicação, 
Tabela 4. Médias, teste de Tukey e coeficientes de variação referentes à produtividade de grãos de trigo em função da adubação nitrogenada

\begin{tabular}{lcc}
\hline \multicolumn{1}{c}{ Anos } & Produtividade de grãos de trigo $\left.\mathbf{~ ( k g ~ h a ~}^{-1}\right)$ \\
\hline Cultivares & $\mathbf{2 0 0 6}$ & $\mathbf{2 0 0 7}$ \\
E 21 & & \\
IAC 370 & $3657 \mathrm{a}$ & $3605 \mathrm{a}$ \\
DMS (5\%) & $3740 \mathrm{a}$ & $3700 \mathrm{a}$ \\
\hline Fontes de N & 206 & 109 \\
Entec & & \\
Sulfato de amônio & $3585 \mathrm{a}$ & $3612 \mathrm{a}$ \\
Uréia & $3533 \mathrm{a}$ & $3648 \mathrm{a}$ \\
DMS (5\%) & $3567 \mathrm{a}$ & $3696 \mathrm{a}$ \\
\hline Épocas de aplicação & 163 & 160 \\
Semeadura & & \\
Cobertura & $3574 \mathrm{a}$ & $3606 \mathrm{a}$ \\
DMS (5\%) & $3549 \mathrm{a}$ & $3698 \mathrm{a}$ \\
\hline Doses de N (kg ha-1) & 111 & 109 \\
0 & & \\
50 & 3029 & 2537 \\
100 & 3755 & 4164 \\
150 & 3791 & 4021 \\
200 & 3690 & 3858 \\
Média geral & 3543 & 3680 \\
CV (\%) & 3562 & 3652 \\
\hline
\end{tabular}

Médias seguidas de letras iguais, na coluna, não diferem entre si pelo teste de Tukey, em nível de 0,05 de probabilidade

foi efetuada a irrigação no experimento reduzindo, então, as perdas por volatilização, principalmente da ureia. Comparações entre diversas fontes de fertilizantes nitrogenados foram feitas por vários autores, em geral em condições satisfatórias de umidade do solo (próximo da capacidade de campo), não têm sido encontradas diferenças na eficiência dessas fontes para a produtividade de grãos de trigo no Cerrado, em função da baixa lixiviação de nitrogênio. Megda et al. (2009) avaliaram as fontes Entec, sulfato de amônio e ureia, e Silva et al. (2008), a ureia e o Entec em sistema plantio direto. Nenhum desses autores verificou efeito das fontes sobre a produtividade em detrimento das semelhantes condições edafoclimáticas.

Com relação às doses de $\mathrm{N}$ (Figura 3), elas influenciaram a produtividade de grãos de trigo em 2006 e 2007 e se ajustaram às funções quadráticas, com a máxima produtividade sendo alcançada, respectivamente, com a estimativa de 118 e 119 $\mathrm{kg} \mathrm{ha}^{-1}$ de N. Também Zagonel et al. (2002), Trindade et al. (2006) e Teixeira Filho et al. (2009) verificaram efeito do N na produtividade da cultura. Por outro lado, Silva (1991), devido ao fornecimento de $\mathrm{N}$ pela cultura da soja cultivada ha vários anos na área e Pottker et al. (1984), em função das condições climáticas adversas (altas precipitações pluviais alternadas com períodos de seca), não verificaram efeito da aplicação de $\mathrm{N}$ na produtividade da cultura.

A Figura 3 mostra o modelo de regressão desenvolvido com os valores médios de produção em função das doses de nitrogênio, conforme Tabela 4. Verifica-se que o valor do coeficiente de determinação da reta $\left(\mathrm{R}^{2}\right)$ do ano de 2007 não foi tão alto, o que indica que pode haver ajuste a funções que possibilitam uma estimativa melhor da dose de $\mathrm{N}$ que

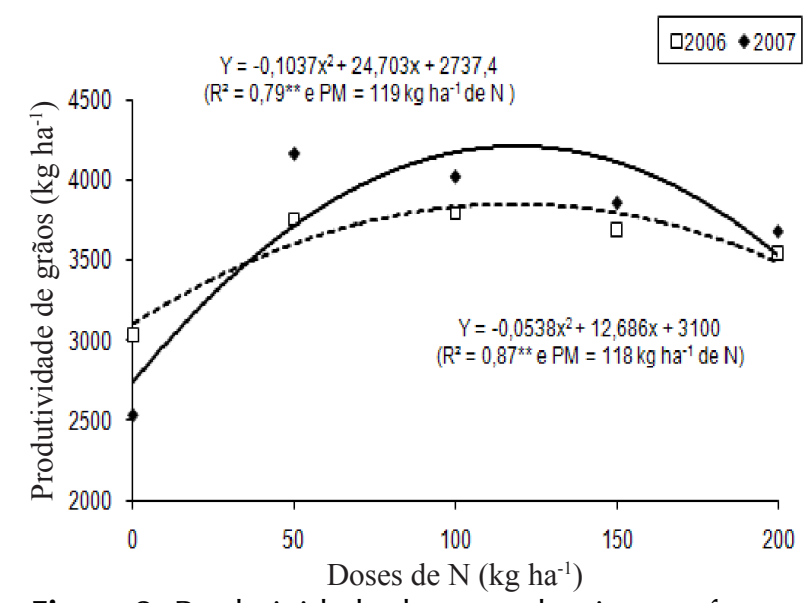

Figura 3. Produtividade de grãos de trigo em função de doses de N, nas safras 2006 e 2007

proporciona a máxima produtividade de grãos de trigo. Ressaltase que nem sempre o $\mathrm{R}^{2}$ será melhor ou pior apenas por uma questão do coeficiente de variação (CV\%) dos resultados, o que pode ser observado pelo $\mathrm{CV} \%$ da produtividade de grãos de 2006 que foi maior em relação ao ano de 2007 (Tabela 4) enquanto, para esta mesma avaliação, o $\mathrm{R}^{2}$ de 2006 foi maior (melhor) quando comparado com o ajuste quadrático da produtividade de 2007 (Figura 3).

Os resultados encontrados em relação à modelagem neuro fuzzy mostraram que é uma técnica apropriada para estimar a produtividade do trigo em função da adubação nitrogenada, considerando os valores das doses de $\mathrm{N}\left(\mathrm{kg} \mathrm{ha}^{-1}: 0,50,100,150\right.$, 200) e desempenho de saída satisfatório.

As Figuras $4 \mathrm{~A}$ e $4 \mathrm{~B}$ evidenciaram os valores de produção estimados nos anos de 2006 e 2007, respectivamente, pelo modelo de predição do ANFIS.

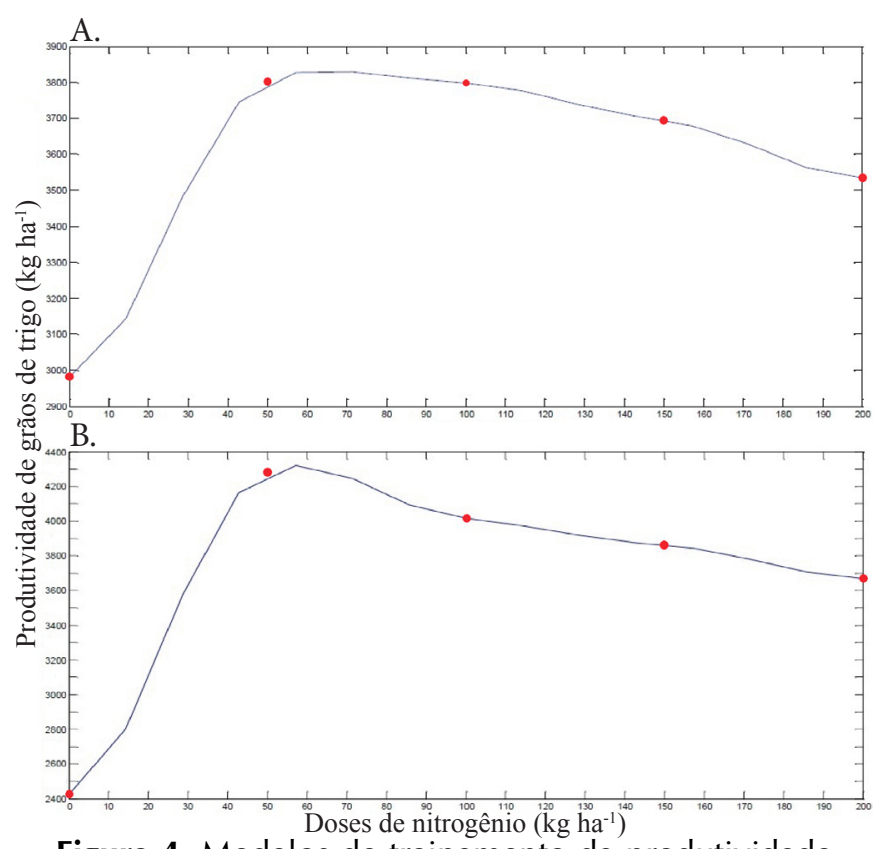

Figura 4. Modelos de treinamento da produtividade de grãos de trigo no ANFIS, dos anos de 2006 (A) e de 2007 (B) em função das doses de nitrogênio 
Após o treinamento da rede neural a raiz quadrada do erro médio quadrático (RMSE) dos anos de 2006 e 2007 (Tabela 5) foi menor que o RMSE calculado com o modelo de regressão com aproximação quadrática. O valor do erro RMSE mostra que a aprendizagem para predição da produção de trigo foi satisfatória para o modelo ANFIS, sendo possível obter-se a estimação, baseada em dados experimentais da cultura do trigo com boa precisão.

Tabela 5. Valor comparativo do RMSE dos modelos de regressão quadrática e ANFIS

\begin{tabular}{ccc}
\hline \multicolumn{3}{c}{ RMSE } \\
\hline Ano da produção & Regressão quadrática & ANFIS \\
2006 & 94,36 & 30,19 \\
2007 & 265,14 & 71,76 \\
\hline
\end{tabular}

Na Tabela 6 constam os dados de saída de produtividade de trigo estimados em função das doses de $\mathrm{N}\left(\mathrm{kg} \mathrm{ha}^{-1}\right)$, em valores médios de produção, no modelo de regressão quadrática e no modelo ANFIS demonstrando, no geral, valores muito próximos de produtividade de grãos para todas as doses de $\mathrm{N}$, tanto na safra 2006 como na 2007.

Tabela 6. Valores de produtividade de grãos de trigo gerados no ANFIS, regressão quadrática e valores médios de produção real por dose de $\mathrm{N}$

\begin{tabular}{lcccccc}
\hline \multirow{2}{*}{ Modelo gerado } & Safra & \multicolumn{5}{c}{$\begin{array}{c}\text { Produtividade de grãos } \\
\text { de trigo (kg ha-1) }\end{array}$} \\
\cline { 3 - 7 } & & $\mathbf{0}$ & $\mathbf{5 0}$ & $\mathbf{1 0 0}$ & $\mathbf{1 5 0}$ & $\mathbf{2 0 0}$ \\
Valor médio & 2006 & 3029 & 3755 & 3791 & 3690 & 3543 \\
de produção real & 2007 & 2537 & 4164 & 4021 & 3858 & 3680 \\
ANFIS & 2006 & 2980 & 3800 & 3796 & 3693 & 3533 \\
& 2007 & 2427 & 4280 & 4014 & 3860 & 3668 \\
Regressão quadrática & 2006 & 3100 & 3600 & 3831 & 3792 & 3485 \\
& 2007 & 2737 & 3713 & 4171 & 4110 & 3530 \\
\hline
\end{tabular}

O ANFIS combina a capacidade de aprendizado das redes neurais com o poder de interpretação linguística da lógica fuzzy. Para uma rede neural aprender, fazem-se necessários dados experimentais anteriores. Portanto, a maior quantidade de dados experimentais, assim como a experimentação por período de tempo maior, tende a proporcionar melhores resultados com o ANFIS, uma vez que o aprendizado do ANFIS será mais eficiente. Assim, pode-se minimizar prováveis efeitos de fatores externos aos avaliados no experimento, os quais poderiam ser responsáveis pela variação na produtividade.

\section{Conclusões}

1. O estudo mostra que o ANFIS pode ser utilizado para desenvolver um modelo híbrido de estimação da produtividade de trigo em função da adubação nitrogenada utilizando-se, para isto, os valores médios de produtividade real avaliada durante dois anos, na região de cerrado.

2. O modelo neuro fuzzy gerado possibilitou estimar os valores de produtividade em função das doses de nitrogênio com os valores do erro menores que o RMSE do modelo utilizando regressão quadrática nos anos de 2006 e 2007.
3. O modelo de estimação da produtividade de trigo gerado em função da adubação nitrogenada mostrou-se eficiente quando comparado com o modelo de regressão quadrática, pela precisão dos valores estimados de produtividade do trigo nas doses de $\mathrm{N}$ de $0,50,100,150$ e $200 \mathrm{~kg} \mathrm{ha}^{-1}$.

\section{Agradecimentos}

Os autores agradecem o apoio financeiro da FAPESP, do CNPq e da CAPES.

\section{Literatura Citada}

Alvarez, R. Predicting average regional yield and production of wheat in the Argentine Pampas by an artificial neural network approach. European Journal of Agronomy, v.30, p.70-77, 2009.

Alves, M. C.; Pozzab, E. A.; Costa, J. C. B.; Carvalho, L. G.; Alves, L. S. Adaptative neuro fuzzy inference systems for epidemiological analysis of soybean rust. Enviromental Modeling \& Software, v.26, p.1-8, 2011.

Bucene, L. C.; Rodrigues, L. H. A. Utilização de redes neurais artificiais para a avaliação de produtividade do solo, visando classificação de terras para irrigação. Revista Brasileira de Engenharia Agrícola e Ambiental, v.8, p.326329, 2004.

Carvalho, C. C. N.; Rocha, W. F.; Ucha J. M. Mapa digital de solos: Uma proposta metodológica usando Inferência Fuzzy. Revista Brasileira de Engenharia Agrícola e Ambiental, v.13, p.46-55, 2009.

CQFS-RS/SC - Comissão de Química e Fertilidade do Solo - RS/ SC. Manual de adubação e de calagem para os estados do Rio Grande do Sul e de Santa Catarina. Sociedade Brasileira de Ciência do Solo - Núcleo Regional Sul. Porto Alegre, 2004, 394p.

Ferreira, D. F. SISVAR: Um programa para análises e ensino de estatística. Revista Symposium, v.6, p.36-41, 2008.

Ghoush, M. A.; Samhouri, M.; Al-Holy, M.; Herald, T. Formulation and fuzzy modeling of emulsion stability and viscosity of a gum-protein emulsifier in a model mayonnaise system. Journal of Food Engineering, v.84, p.348-357, 2008.

Huang, Y.; Lan, Y.; Thomson S. J.; Fang A.; Hoffmann W. C.; Lacey, R. E. Development of soft computing and applications in agricultural and biological engineering. Computers and Eletronics in Agriculture, v.71, p.107-127, 2010.

Jang, J. S. R. ANFIS: Adaptive-network-based fuzzy inference system. IEEE Transactions on Systems, Man and Cybernetics, v.23, p.665-685, 1993.

Jang, J. S. R.; Sun C. T. Neuro fuzzy modeling and control. Proceeding of IEEE, v.83, p.378-406, 1995.

Kashaninejad, M.; Dehghani, A. A.; Kashiri, M. Modeling of wheat sing two artificial neural networks (MLP anda RBF). Journal of Food Engineering, v.91, p.602-607, 2009. 
Megda, M. M.; Buzetti, S.; Andreotti, M.; Teixeira Filho, M. M. C.; Vieira, M. X. Resposta de cultivares de trigo ao nitrogênio em relação às fontes e épocas de aplicação sob plantio direto e irrigação por aspersão. Ciência e Agrotecnologia, v.33, p.1055-1060, 2009.

Pottker, D.; Fabrício, A. C.; Nakayama, L. H. I. Doses e métodos de aplicação de nitrogênio para a cultura do trigo. Pesquisa Agropecuária Brasileira, v.19, p.1197-1201, 1984.

RCCBPT - Reunião da Comissão Centro Brasileira de Pesquisa de Trigo, 13., 2004, Goiânia. Informações técnica para a cultura de trigo na região do Brasil Central: safras 2005 e 2006. Santo Antônio de Goiás: Embrapa Arroz e Feijão, 2005. 86p. Documentos, 173

Ros, C. O. da; Salet R. L.; Porn, R. L.; Machado, J. N. C. Disponibilidade de nitrogênio e produtividade de milho e trigo com diferentes métodos de adubação nitrogenada no sistema plantio direto. Ciência Rural, v.33, p.799-804, 2003.

Santos, H. G. dos; Jacomine, P. K. T.; Anjos, L. H. C. dos; Oliveira, V. A. de; Oliveira, J. B. de; Coelho, M. R.; Lumbreras, J. F.; Cunha, T. J. F. (Ed.). Sistema brasileiro de classificação de solos. 2.ed. Rio de Janeiro: Embrapa Solos, 2006. 306p.

Schultz, A.; Wieland R. The use of neural networks in agroecological modeling. Computers and Eletronics in Agriculture, v.18, p.73-90, 1997.

Silva, D. B. Efeito do nitrogênio em cobertura sobre o trigo em sucessão a soja na região dos cerrados. Pesquisa Agropecuária Brasileira, v.26, p.1387-1392, 1991.

Silva, I. N.; Spatti, S. H.; Flauzino R. A. Redes neurais artificiais para engenharia e ciências aplicadas. São Paulo: Artliber, 2010. 399p.

Silva, S. A. da; Arf, O.; Buzetti, S.; Silva, M. G. da. Fontes e épocas de aplicação de nitrogênio em trigo em sistema plantio direto no Cerrado. Revista Brasileira de Ciência do Solo, v.32, p.2717-2722, 2008.
Tanscheit, R.; Gomide, F. A. C.; Teixeira, M. C. M. Modelagem e controle nebuloso. In: L. A. Aguirre (org). Enciclopédia de Automática. 1.ed. São Paulo: Edgar Blucher, 2007. v.3, p.283-323.

Teixeira Filho, M. C. M.; Buzetti, S.; Andreotti, M.; Arf, O.; Benett, C. G. S. Doses, fontes e épocas de aplicação de nitrogênio em trigo irrigado em plantio direto. Pesquisa Agropecuária Brasileira, v.45, p.797-804, 2010.

Teixeira Filho, M. C. M.; Buzetti, S.; Andreotti, M.; Sá, M. E. de; Arf, O.; Megda, M. M. Response of irrigated wheat cultivars to different nitrogen rates and sources. Revista Brasileira de Ciência do Solo, v.33, p.1303-1310, 2009.

Trindade, M. da G.; Stone, L. F.; Heinemann, A. B.; Cánovas, A. D.; Moreira, J. A. A. Nitrogênio e água como fatores de produtividade do trigo no Cerrado. Revista Brasileira de Engenharia Agrícola e Ambiental, v.10, p.24-29, 2006.

van Raij, B.; Andrade, J. C.; Cantarella, H.; Quaggio, J. A. Análise química para avaliação da fertilidade de solos tropicais. Campinas: IAC, 2001. 285p.

Yilmaz I.; Kaynar O. Multiple regression, ANN (RBF, MLP) and ANFIS models for prediction of swell potencial of clayey soils. Expert systems with applications, v.38, p.5958-5966, 2011.

Zadeh L. A. Fuzzy sets. Information and Control, v.8, p.338353, 1965.

Zadeh L. A. Fuzzy Algorithms. Information and control, v.12, p.94-102, 1968.

Zagonel, J.; Venâncio, W. S.; Kunz, R. P.; Tanamati, H. Doses de nitrogênio e densidade de plantas com e sem um regulador de crescimento afetando o trigo, cultivar OR-1. Ciência Rural, v.32, p.25-29, 2002.

Zamudio, M. A. M.; Pérez A., López F.; Garcia, J. C.; Feria M. J.; Alfaro, A. Neural fuzzy model applies to autohydrolysis of Paulownia trihybrid. Journal of the Taiwan Institute of Chemical Engineers, v.42, p.292-297, 2011. 